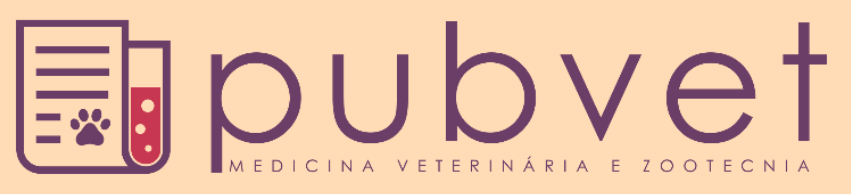

https://doi.org/10.31533/pubvet.v13n01 a243.1-7

\title{
Frequência da tipagem sanguínea em felinos domésticos atendidos em uma clínica veterinária em Dourados, Mato Grosso do Sul, Brasil
}

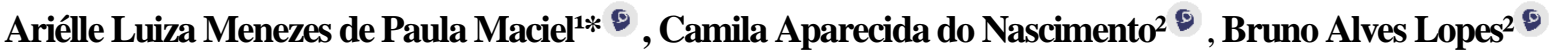 \\ ${ }^{I}$ Acadêmica do curso de Medicina Veterinária do Centro Universitário da Grande Dourados (UNIGRAN). Dourados - MS, Brasil. \\ ${ }^{2}$ Médico(a) Veterinária da Clínica Veterinária da UNIGRAN, Dourados, Mato Grosso do Sul, Brasil \\ *Autor para correspondência, E-mail: arielle.luiza@hotmail.com
}

\begin{abstract}
RESUMO. Este trabalho teve como objetivo determinar a frequência dos tipos sanguíneos de felinos domésticos atendidos na Clínica Veterinária do Centro Universitário da Grande Dourados (UNIGRAN), na cidade de Dourados, Mato Grosso do Sul. Os felinos possuem tipos sanguíneos semelhantes ao sistema ABO dos humanos, porém sem a existência do fator Rh. Os grupos sanguíneos dos felinos são constituídos por tipo A (mais frequente), tipo B (presente em algumas raças puras) ou tipo AB (raro). Para essa pesquisa, foram selecionados 50 animais, machos e fêmeas, acima de dois meses de idade, independente de peso e raça. As amostras de sangue foram coletadas através de venopunção da jugular e a tipagem sanguínea foi realizada através do kit comercial RapidVet-H IC Feline Immuno-Chromatographic (DMS Laboratories, Flemington, NJ, USA). O presente trabalho obteve frequência de $100 \%$ de felinos domésticos com tipo A. Não foram encontrados gatos do tipo B e AB. Os resultados encontrados neste estudo indicam que na região de Dourados, há uma frequência maior de gatos mestiços do grupo sanguíneo A. A tipagem sanguínea é um exame primordial antes de realizar qualquer tipo de procedimento hemoterápico, pois evita a ocorrência de reação transfusional com tipo sanguíneo incompatível (tanto para doador quanto para receptor), visto que os animais podem vir a óbito em situação de incompatibilidade sanguínea.
\end{abstract}

Palavras chave: gatos, medicina transfusional, tipo sanguíneo, sistema $\mathrm{AB}$ felino

\section{Frequency of blood typing in domestic cats treated at a veterinary clinic in Dourados, Mato Grosso do Sul, Brazil}

ABSTRACT. The objective of this study was to determine the frequency of blood types
of domestic cats treated at the Veterinary Clinic of the University Center of Grande
Dourados (UNIGRAN), in the city of Dourados, Mato Grosso do Sul. Felines have
blood types similar to the ABO system of humans, but without the existence of the Rh
factor. The blood groups of the felines consist of type A (more frequent), type B
(present in some pure races) or type AB (rare). For this research, we selected 50 male
and female animals, aged from two months to thirteen years, regardless of weight and
race. Blood samples were collected by jugular venipuncture and blood typing was
performed using the RapidVet-H commercial kit Feline Immuno-Chromatographic
(DMS Laboratories, Flemington, NJ, USA). The present study obtained a frequency of
$100 \%$ of domestic felines with type A. No type B and AB cats were found. The results
found in this study indicate that in the region of Dourados, there is a higher frequency
of crossbred cats of the blood group A. The blood typing is a primordial examination
before performing any type of hemotherapy procedure, since it avoids the occurrence 
of transfusional reaction with sanguine type incompatible (both for donor and recipient), since the animals can die in a situation of blood incompatibility.

Keywords: cats, transfusion medicine, blood type, $\mathrm{AB}$ feline system

\title{
Frecuencia de la tipificación sanguínea de los felinos domésticos atendidos en una clínica veterinaria de Dourados, Mato Grosso do Sul, Brasil
}

\begin{abstract}
RESUMEN. Este trabajo tuvo como objetivo determinar la frecuencia de los tipos sanguíneos de felinos domésticos atendidos en la Clínica Veterinaria del Centro Universitario de la Grande Dourados (UNIGRAN), en la ciudad de Dourados, Mato Grosso do Sul. Los felinos poseen tipos sanguíneos semejantes al sistema ABO de los humanos, pero sin la existencia del factor Rh. Los grupos sanguíneos de los felinos están constituidos por tipo A (más frecuente), tipo $\mathrm{B}$ (presente en algunas razas puras) o tipo $\mathrm{AB}$ (raro). Para esta investigación, se seleccionaron 50 animales, machos y hembras, con edad entre dos meses a trece años, independientemente de peso y raza. Las muestras de sangre fueron colectadas a través de venopunción de la yugular y la tipificación sanguínea fue realizada a través del kit comercial RapidVet-H IC Feline Immuno-Chromatographic (DMS Laboratories, Flemington, NJ, USA). El presente trabajo obtuvo una frecuencia de $100 \%$ de felinos domésticos con tipo A. No se encontraron gatos del tipo B y AB. Los resultados encontrados en este estudio indican que en la región de Dourados hay una frecuencia mayor de gatos mestizos del grupo sanguíneo A. La tipificación sanguínea es un examen primordial antes de realizar cualquier tipo de procedimiento hemoterapéutico pues evita la incidencia de la reacción transfusional con tipo sanguíneo incompatible (tanto para donante como para receptor), ya que los animales pueden fallecer en situación de incompatibilidad sanguínea.
\end{abstract}

Palabras clave: gatos, medicina transfusional, tipo sanguíneo, sistema AB felino

\section{Introdução}

Os grupos sanguíneos possuem uma grande importância na área da hemoterapia em qualquer espécie animal, a fim de evitar reações transfusionais. Em felinos, a tipagem sanguínea é essencial para se evitar a isoeritrólise neonatal (IN) e reações hemolíticas agudas pós-transfusionais (Pinto et al, 2016), visto que a transfusão sanguínea é um procedimento frequentemente utilizado na clínica médica veterinária em felinos domésticos (Barfield \& Adamantos 2011).

Os gatos possuem três tipos sanguíneos: A, B e AB, variando conforme raça e localização geográfica. O grupo A é o mais frequente, encontrado principalmente em mestiços, chegando a ter $85 \%$ a $100 \%$ de frequência em alguns países (Thrall 2015), enquanto que o grupo B é mais comum de ser identificado em gatos de raças puras (Persas, Devon Rex, British Shorthair) (Fosset \& Blais 2014). Já o tipo AB raramente é descrito (Giger et al, 1991). Embora sejam aplicadas as letras iguais aos tipos sanguíneos humanos, não há relação sorológica alguma entre o sistema $\mathrm{AB}$ felino e o sistema $\mathrm{ABO}$ humano (Lacerda et al, 2008). Os tipos sanguíneos do sistema AB felinos, são determinados por pelo menos dois alelos (A, b) no mesmo locus. O alelo tipo A parece ser completamente dominante sobre o alelo b. Por isso, gatos com fenótipo A podem ser homozigotos ou heterozigotos ( $\mathrm{A} / \mathrm{A}$ ou $\mathrm{A} / \mathrm{b})$, enquanto que gatos homozigotos para o alelo B (B/B) expressam quantidades suficientes do antígeno eritrocitário $\mathrm{B}$, mas não possuem antígeno $\mathrm{A}$. Já o terceiro alelo $\mathrm{AB}$, aparenta ser recessivo em relação ao alelo $\mathrm{A}$, mas dominante sobre o alelo B, determinando a herança do tipo sanguíneo AB (Lacerda et al, 2008; Sorgatto et al, 2017).

A alta ocorrência de reações hemolíticas graves na primeira transfusão ocorre, pois, os gatos, diferentemente dos cães, possuem haloanticorpos (conhecidos também como isoanticorpos), ou seja, anticorpos naturais contra antígenos de eritrócitos de sangue que pertencem a um tipo sanguíneo diferente do seu. Esses haloanticorpos possuem grande importância na clínica de felinos, pois são eles que condicionam a gravidade e severidade das reações transfusionais e da isoeritrólise neonatal (IN) (Knottenbelt et al, 1999; Fragío et al, 2009; Sorgatto et al, 2017). 
Os gatos do tipo B, com mais de 3 meses de idade, possuem grande quantidade de anticorpos antiA, da classe IgM., que são anticorpos responsáveis pelas reações de hipersensibilidade do tipo II, que se caracterizam por serem potencialmente fatais, pois podem resultar em reações transfusionais hemolíticas agudas graves, bem como isoeritrólise neonatal (doença hemolítica do recém-nascido). Enquanto isso, os gatos do tipo A, possuem de 35\% a 44\% anticorpos detectáveis anti-B. Estes anticorpos anti-B (IgM e IgG) têm atividade aglutinante e hemolítica muito menor que o anti-A, e são responsáveis por reações transfusionais moderadas. Gatos tipo AB não possuem anticorpos naturais, pois expressam os dois antígenos (Weinstein et al, 2007; Fosset \& Blais 2014).

A IN ocorre quando um filhote do tipo sanguíneo A ou AB nasce de uma fêmea do tipo B (Andrade 2008). Na ingestão do colostro, são ingeridos altos níveis de anticorpos anti-A adquiridos da mãe, e estes são absorvidos nas primeiras dezesseis horas de vida, ocasionando a destruição das hemácias do neonato (August 2001). Os sinais clínicos da IN podem variar de agudos a sub-clínicos e incluem anemia, hemoglobinemia, hemoglobinúria, icterícia, deficiência no desenvolvimento, necrose na ponta da cauda e até morte súbita (Feldman 1997; Aubert et al, 2011; Nelson \& Couto 2015).

As reações transfusionais observadas durante a administração de sangue em felinos do tipo A que recebem sangue do tipo B, caracterizam-se por sinais clínicos como febre, vômitos, vocalização, letargia, icterícia, hipotensão e morte. Mas para os felinos do tipo B que recebem sangue tipo A, apenas alguns mililitros de sangue podem ser suficientes para causar a morte do animal. Felinos com tipo $\mathrm{AB}$, teoricamente poderiam receber sangue tanto do tipo A quanto do tipo $\mathrm{B}$. Mas na prática, receber sangue tipo $\mathrm{B}$ não é possível, pois a quantidade de anticorpos anti-A presente neste tipo sangue, pode acarretar reação transfusional grave. Por isso na ausência de um doador AB, é indicado que esse animal receba sangue somente do tipo A. Mas o ideal sempre é receber a transfusão de um gato do mesmo tipo sanguíneo (Lanevschi \& Wardrop 2001).

Esste trabalho teve como objetivo classificar e determinar a frequência e prevalência dos tipos sanguíneos de felinos atendidos na Clínica Veterinária do Centro Universitário da Grande Dourados (UNIGRAN), na cidade de Dourados, Mato Grosso do Sul, sabendo que é essencial realizar a tipificação sanguínea antes de qualquer procedimento hemoterápico, dado que em caso de incompatibilidade, acarretará sérios problemas orgânicos de caráter fatal, como reações transfusionais hemolíticas imunomediadas ou isoeritrólise neonatal (doença hemolítica do recém-nascido).

\section{Material e métodos}

O presente trabalho foi submetido à Comissão de Ética na Utilização de Animais (CEUA) da UNIGRAN e aprovado sob protocolo de número: 019/2017. Foram utilizados 50 gatos, machos e fêmeas, a partir de dois meses, independente de raça e peso, abordados no ato da consulta ambulatorial na Clínica Veterinária da UNIGRAN, sob autorização prévia dos proprietários e que eram provenientes do município de Dourados, MS.

Após tricotomia e assepsia adequada, foram coletadas de cada animal amostras de sangue $(2-3 \mathrm{~mL})$ por punção de veia jugular, sendo colocadas em tubos contendo ácido etilenodiaminotetracético (EDTA). A tipagem foi efetuada por meio de kits comerciais RapidVet-H IC Feline ImmunoChromatographic (DMS Laboratories, Flemington, NJ,USA) de acordo com as instruções do fabricante, sendo utilizado um cartão para cada animal. A técnica consistiu em pipetar 1 gota do sangue armazenado no tubo com EDTA, dentro do microtubo pré-cheio com diluente, para preparar a diluição do sangue, invertendo suavemente o frasco várias vezes. Com o dispositivo em superfície plana, instilou-se 3 gotas do sangue diluído na porta de amostra redonda e após aguardar alguns segundos a absorção da amostra, foram instiladas 3 gotas do frasco conta-gotas com tampão especial na mesma abertura.

Foram aguardados de 5-10 minutos para visualização das linhas de resultados. Um resultado positivo é apresentado apenas pela formação de uma linha vermelha vertical em um fundo branco e a janela de controle deverá mostrar uma linha vermelha horizontal, indicando que o teste foi executado corretamente (Figura 1). 


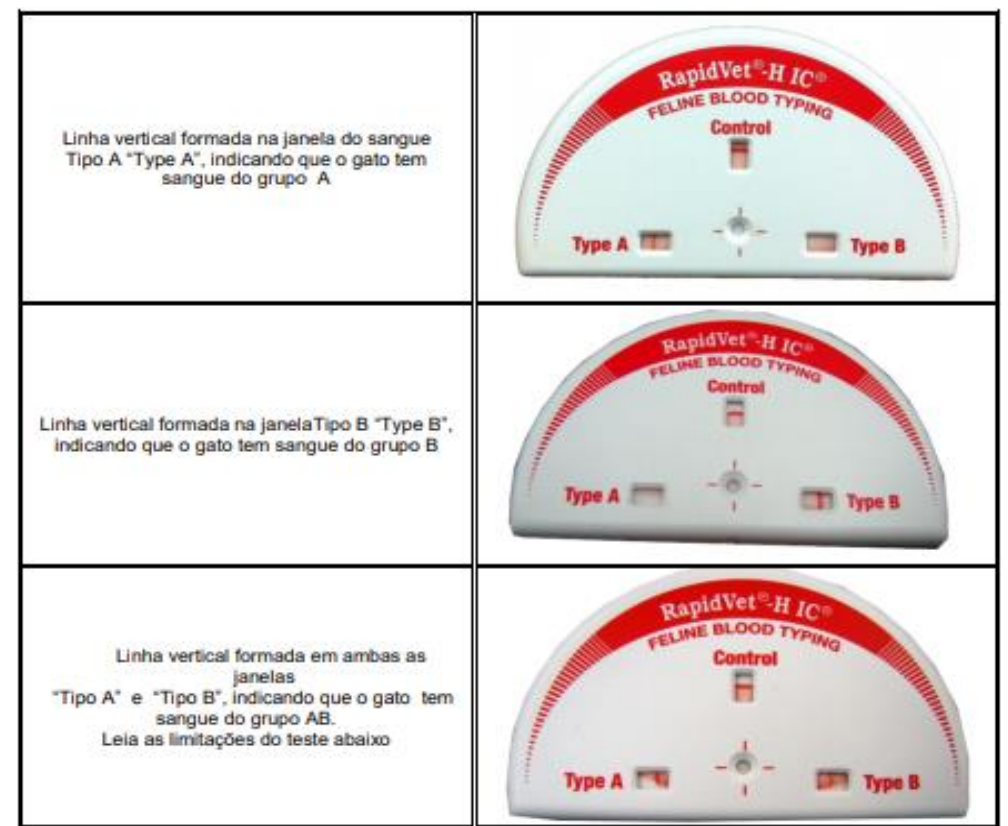

Figura 1. Interpretação dos resultados do teste RapidVet-H IC Feline (DMS Laboratories, Flemington, NJ, USA)

Todos os dispositivos de teste foram identificados na parte da frente com o nome do animal/identificação do gato e a data da realização da tipagem sanguínea (Figura 2).

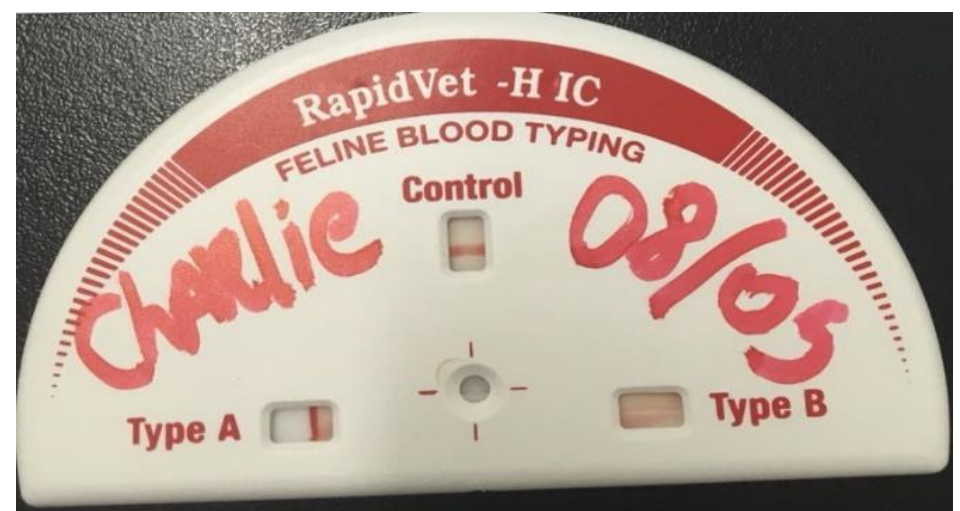

Figura 2. Dispositivo de teste identificado com o nome do animal e a data da realização da tipagem

Os dados coletados foram transformados em dados relativos (porcentagens) e os resultados obtidos representados por meio de gráficos e tabelas, com o propósito de obter principalmente, a frequência dos tipos sanguíneos em percentuais, identificando dentro da população testada, a proporção existente entre os tipos sanguíneos.

\section{Resultados e discussão}

Dos 50 felinos avaliados, 47 eram sem raça definida (SRD), 2 da raça Persa e 1 da raça Angorá. Em relação ao sexo, 44\% (22/50) eram fêmeas e 56\% (28/50) eram machos. Todos os animais avaliados foram positivos para o tipo sanguíneo A, obtendo então a frequência de $100 \%$ dos animais para esse grupo. Neste estudo não foram encontrados gatos com tipo B e AB. Na Tabela 1 está descrito a frequência do sistema $\mathrm{AB}$, conforme a raça de cada animal.

Os achados observados nesse estudo são semelhantes aos observados em pesquisas realizada na Finlândia por Giger et al, (1992), onde todos os animais foram do tipo A e não foram encontrados tipo sanguíneo B e AB na população de gatos estudada. Em outros trabalhos, a ausência de B ou AB também foram descritas (Giger et al, 1991; Hubler et al, 1993; Hirota et al, 1995; Souza 1998; Knottenbelt 2002; Vilar 2006; Lacerda et al, 2008; Sorgatto et al, 2017). 
Tabela 1. Frequência da tipagem sanguínea de acordo com a raça

\begin{tabular}{lcccc}
\hline Raça & $\mathrm{N}$ & Tipo A (\%) & Tipo B (\%) & Tipo C (\%) \\
\hline SRD & 47 & $94 \%$ & $0 \%$ & $0 \%$ \\
Persa & 2 & $4 \%$ & $0 \%$ & $0 \%$ \\
Angorá & 1 & $2 \%$ & $0 \%$ & $0 \%$ \\
Total & 50 & $100 \%$ & $0 \%$ & $0 \%$ \\
\hline
\end{tabular}

$\mathrm{N}$ = população dos felinos avaliados; $\mathrm{SRD}=$ sem raça definida; \%= frequência relativa.

Ainda há poucos estudos sobre a frequência dos tipos sanguíneos dos gatos, realizados no Brasil (Tabela 2). Nos demais artigos conduzidos e publicados no país, os resultados também foram semelhantes e mostraram a predominância do grupo sanguíneo A em relação aos outros grupos (Souza (Souza 1998; Vilar 2006; Guerra et al, 2007; Lacerda et al, 2008; Medeiros et al, 2008; Mendes et al, 2013; Pinto et al, 2016; Silva et al, 2016; Sorgatto et al, 2017).

Tabela 2. Frequência dos tipos sanguíneos de felinos domésticos em algumas regiões do Brasil

\begin{tabular}{|c|c|c|c|c|c|c|}
\hline \multirow{2}{*}{ Região } & \multirow{2}{*}{ Cidade } & \multirow{2}{*}{$\mathrm{N}$} & \multicolumn{3}{|c|}{ Frequência dos tipos sanguíneos } & \multirow{2}{*}{ Referências } \\
\hline & & & Tipo A (\%) & Tipo B (\%) & Tipo AB (\%) & \\
\hline \multirow[t]{2}{*}{ Sul } & Porto Alegre & 105 & 97,1 & 1,9 & 1,0 & Guerra et al, (2007) \\
\hline & Porto Alegre & 100 & 97,0 & 3,0 & 0,0 & Lacerda et al, (2008) \\
\hline \multirow[t]{3}{*}{ Sudeste } & Rio de Janeiro & 48 & 93,75 & 0,0 & 6,25 & Vilar (2006) \\
\hline & Rio de Janeiro & 172 & 94,8 & 2,9 & 2,3 & Medeiros et al, (2008) \\
\hline & Rio de Janeiro & 86 & 98,8 & 1,2 & 0,0 & Pinto et al, (2016) \\
\hline \multirow[t]{2}{*}{ Nordeste } & Paraíba & 164 & 98,1 & 1,21 & 0,69 & Mendes et al, (2013) \\
\hline & Salvador & 58 & 93,3 & 6,7 & 0 & Souza (1998) \\
\hline \multirow[t]{3}{*}{ Centro-oeste } & Goiânia & 15 & 93,33 & 6,66 & 0,0 & Silva et al, (2016) \\
\hline & Campo Grande & 202 & 98,5 & 0,0 & 1,5 & Sorgatto et al, (2017) \\
\hline & Dourados & 50 & 100 & 0,0 & 0,0 & Este Estudo (2018) \\
\hline Norte & Pará & 235 & 98,3 & 0,42 & 1,28 & Silva et al, (2016) \\
\hline
\end{tabular}

Adaptada de Sorgatto et al, (2017). N = população de felinos estudados, \%= frequência relativa.

Esta pesquisa difere-se do estudo realizado por Sorgatto et al, (2017) em Campo Grande, MS, onde foram identificados $1,5 \%$ de felinos do tipo sanguíneo $\mathrm{AB}$, mostrando que há uma peculiaridade na população estudada na capital do Estado. Todavia, não há como afirmar se a diferença encontrada é caracterizada pela localização geográfica da cidade, se está relacionada à variação populacional estudada ou se foram testados poucos animais neste estudo, comparado aos de Sorgatto et al, (2017). Esta pesquisa é a terceira realizada na região do Centro-Oeste.

Segundo Knottenbelt (2002), a determinação da distribuição dos tipos sanguíneos da população felina local, pode ajudar a estabelecer o risco de ocorrência de reações transfusionais. Embora o tipo sanguíneo A seja predominante na maioria dos estudos, em alguns países a porcentagem de tipo B e AB são relativamente altos. O tipo B chega a ter um percentual que varia entre $30 \%$ e $60 \%$ em algumas raças, como no Devon Rex, Cornish Rex, Van Turco, Angorá Turco (Lacerda et al, 2008).

No Reino Unido, uma pesquisa realizada com 121 gatos da raça British Shorthair, a frequência do tipo B $(58,7 \%)$ naquela população, ultrapassou a do tipo A $(39,7 \%)$ e ainda teve uma porcentagem de grupo AB (1,6\%) (Knottenbelt et al, 1999).

Nos Estado Unidos, uma pesquisa com 85 animais da raça Birmanesa também obteve uma porcentagem alta de felinos com grupo B $(58,8 \%)$ em relação ao grupo A $(41,2 \%)$ (Giger et al, 1991). Já no Japão, uma pesquisa com 238 gatos, obteve-se uma frequência de $9,2 \%$ do tipo $A B$, considerada uma porcentagem alta em comparação a outros artigos (Ejima et al, 1986). No entanto, devemos lembrar que as estatísticas mudam conforme a região geográfica e as raças. Estudos realizados na Inglaterra por 
Forcada et al, (2007) e Zheng et al, (2011) demonstraram uma prevalência de 11 a 30\% do sangue tipo $\mathrm{B}$ em gatos SRD. Isso nos mostra que a mistura entre gatos mestiços com os de raça pura podem mudar completamente a frequência desses grupos sanguíneos, diferente dos resultados obtidos no presente artigo.

\section{Conclusão}

Esta pesquisa obteve frequência de $100 \%$ de gatos com tipo sanguíneo A. Esse achado permite saber a prevalência do grupo sanguíneo na população estudada e por isso reforça a necessidade da realização da tipagem sanguínea antes de qualquer transfusão.

\section{Agradecimentos}

Os autores agradecem a Distribuidora VP Diagnóstico, pelo fornecimento dos kits RapidVet-H IC Feline Immuno-Chromatographic para realização desta pesquisa.

\section{Referências bibliográficas}

Andrade S.F. (2008) Manual da Terapêutica Veterinária. Rocca, São Paulo.

Aubert I., Abrams-Ogg A.C.G., Sylvestre A.M., Dyson D.H., Allen D.G. \& Johnstone I.B. (2011) The use of vascular access ports for blood collection in feline blood donors. Canadian Journal of Veterinary Research 75, 25-34.

August J.R. (2001) Consultations in Feline Internal Medicine. WB Saunders Company, Philadelphia, USA.

Barfield D. \& Adamantos S. (2011) Feline blood transfusions: a pinker shade of pale. Journal of feline medicine and surgery 13, 11-23.

Ejima h., Kurokawa K. \& Ikemoto S. (1986) Feline red blood cell groups detected by naturally occurring isoantibody. The Japanese Journal of Veterinary Science 48, 971-976.

Feldman E.C. (1997) Tratado de medicina interna veterinária.

Forcada Y., Guitian J. \& Gibson G. (2007) Frequencies of feline blood types at a referral hospital in the south east of England. Journal of Small Animal Practice 48, 570-573.

Fosset F.T.J. \& Blais M.-C. (2014) Prevalence of feline blood groups in the Montreal area of Quebec, Canada. The Canadian Veterinary Journal 55, 1225-1228.

Fragío C., Daza M. \& García E. (2009) Transfusiones sanguíneas en perros y gatos. Clínica veterinaria de pequeños animales 29, 229-238.

Giger U., Bucheler J. \& Patterson D.F. (1991) Frequency and inheritance of A and B blood types in feline breeds of the United States. Journal of Heredity 82, 15-20.

Giger U., Gorman N.T., Hubler M., Leidinger J.I., Leidinger E.F., Lubas G., Niini T. \& Slappendel R.J. (1992) Frequencies of feline A and B blood types in Europe. Animal Genetics 23, 17-18.

Guerra T.A., Lacerda L.A., Oliveira S.T., Esteves V.S. \& Diaz Gonzalez F.H. (2007) Tipagem sanguínea em felinos: 148 gatos domésticos na rotina laboratorial do Lacvet-UFRGS. Acta Scientiae Veterinariae 35, 573-574.

Hirota J., Usui R., Oyamada T. \& Ikemoto S. (1995) The phenotypes and gene frequencies of genetic markers in the blood of Japanese crossbred cats. Journal of Veterinary Medical Science 57, 381-383.

Hubler M., Arnold S., Casal M., Fairburn A., Nussbaumer M. \& Rüsch P. (1993) The blood group distribution in domestic cats in Switzerland. Schweizer Archiv fur Tierheilkunde 135, 231-235.

Knottenbelt C.M. (2002) The feline AB blood group system and its importance in transfusion medicine. Journal of feline medicine and surgery 4, 69-76.

Knottenbelt C.M., Addie D.D., Day M.J. \& Mackin A.J. (1999) Determination of the prevalence of feline blood types in the UK. Journal of Small Animal Practice 40, 115-118.

Lacerda L.A., Oliveira S.T., Guerra T.A., Stein G.G. \& González F.H.D. (2008) Prevalência dos tipos sangüíneos A, B e AB em gatos domésticos mestiços da cidade de Porto Alegre, Rio Grande do Sul, Brasil. Brazilian Journal of Veterinary Research and Animal Science 45, 46-53. 
Lanevschi A. \& Wardrop K.J. (2001) Principles of transfusion medicine in small animals. The Canadian Veterinary Journal 42, 447-454.

Medeiros M.A.S., Soares A.M., Alviano D.S., Ejzemberg R., Silva M.H. \& Almosny N.R. (2008) Frequencies of feline blood types in the Rio de Janeiro area of Brazil. Veterinary clinical pathology 37, 272-276.

Mendes R.S., Gurjão T.A., Souza A.P., Lacerda L.A. \& Silva R.M.N. (2013) Frequência dos antígenos eritrocitários do sistema $\mathrm{AB}$ em felinos domésticos no estado da Paraíba. Pesquisa Veterinária Brasileira 33, 780-784.

Nelson R.W. \& Couto C.G. (2015) Medicina interna de pequenos animais. Elsevier Editora, Amsterdan.

Pinto A.B.T., Silva Medeiros M.A., Brito Jardim M.P. \& Albernaz A.P. (2016) Frequências dos tipos sanguíneos e títulos dos aloanticorpos em felinos domésticos. Ciência Animal Brasileira 17, 225235.

Silva P.B., Monteiro M.V.B., Silva R.R., Albuquerque M.R., Pereira A.C.A., Carreira A.S. \& Monteiro F.O.B. (2016) Frequência dos tipos sanguíneos de gatos oriundos do estado do Pará, Brasil. Acta Veterinaria Brasilica 10, 378-382.

Sorgatto S., Oliveira B.B., Silva Godoy K.C., Antunes T.R., Lacerda L.A. \& Souza A.I. (2017) Frequência dos tipos sanguíneos de gatos domésticos mestiços no município de Campo Grande, Mato Grosso do Sul, Brasil. Medicina Veterinária 11, 172-178.

Souza V.M.M. (1998) Tipagem sanguínea em felinos no município de Salvador. In: Escola de Medicina Veterinária, p. 22 p. Universidade Federal da Bahia, Salvador, Bahia, Brasil.

Thrall M.A. (2015) Hematologia e Bioquímica Clínica Veterinária. Editora Roca, São Paulo, Brasil.

Vilar T.D. (2006) Tipagem sanguínea em gatos-Estudo da prevalência dos antígenos eritrocitários do sistema AB felino utilizando metodologia gelteste no estado do Rio de Janeiro, Brasil. Nosso Clínico $\mathbf{5 4}, 31$.

Weinstein N.M., Blais M.C., Harris K., Oakley D.A., Aronson L.R. \& Giger U. (2007) A newly recognized blood group in domestic shorthair cats: the Mik red cell antigen. Journal of veterinary internal medicine 21, 287-292.

Zheng L., Zhong Y., Shi Z. \& Giger U. (2011) Frequencies of blood types A, B, and AB in non-pedigree domestic cats in Beijing. Veterinary clinical pathology 40, 513-517.

Recebido: 5 novembro, 2018

Aprovado: 26 novembro, 2018

Publicado: 31 dezembro, 2018.

Licenciamento: Este artigo é publicado na modalidade Acesso Aberto sob a licença Creative Commons Atribuição 4.0 (CC-BY 4.0), a qual permite uso irrestrito, distribuição, reprodução em qualquer meio, desde que o autor e a fonte sejam devidamente creditados. 